\title{
Comparação da Eficiência do Treinamento em Entubação Orotraqueal com Vídeo Educacional versus Checklist
}

\section{Comparing the Efficiency of Training in Orotracheal Intubation with Educational Video Versus Checklist}

\author{
Allan Danek \\ Felipe Teles de Arruda \\ Ana Paula Quilici ${ }^{1}$
}

\section{PALAVRAS-CHAVE}

- Medicina;

- Vídeos Educacionais;

- Simulação;

- Entubação Orotraqueal;

- Educação Médica.

\section{KEYWORDS}

- Medicine;

- Educational Videos;

- Simulation;

- Tracheal Intubation;

- Medical Education.
Recebido em: 20/06/2014

Reencaminhado em: 04/11/2015

Aprovado em: 05/04/2016

\section{RESUMO}

O artigo relata uma experiência de desenvolvimento, por estudantes de Medicina, de vídeos educacionais que visam aprimorar o aprendizado e fortalecer o embasamento teórico e prático da entubação orotraqueal. Objetivo: O objetivo do estudo é avaliar a retenção do aprendizado dos alunos da graduação por meio do uso de vídeos no ensino de procedimentos médicos no curso de Medicina. Métodos: Foram avaliados 30 alunos da graduação do curso de Medicina, randomizados em dois grupos de estudo, um com vídeo e outro com material teórico (checklist), para estudo individualizado do tema de entubação orotraqueal. Resultados: Todos os alunos melhoraram o conhecimento teórico e prático do procedimento de entubação orotraqueal $(p<0,00)$. Não houve diferença entre a execução do procedimento entre os grupos ( $p=1)$. Quanto ao conhecimento teórico, o grupo de estudo com vídeos apresentou melhor desempenho na avaliação teórica $(p<0,041)$. Conclusão: As duas ferramentas pedagógicas forneceram ganho de conhecimento a aluno, sendo que o conteúdo teórico do procedimento foi superior no grupo de estudo com vídeos, mas o preparo e a execução do procedimento foram semelhantes entre os grupos.

\section{ABSTRACT}

The paper reports on an experience by medical students to develop educational videos which aim to improve learning and strengthen the theoretical and practical foundations of orotracheal intubation. Objective: The objective of this study is to evaluate the learning retention of undergraduate medical students through the use of educational videos containing medical procedures. Methods: 30 undergraduate medical students were randomized in two study groups, one received educational videos and other received theoretical material (checklist). A comparison was made to evaluate and compare their learning in relation to the orotracheal intubation procedure. Results: All the students demonstrated improved practical and theoretical knowledge of the orotracheal intubation procedure. $(p<0.00)$, no difference was found in the procedure execution between the groups $(p=1)$ and, regarding theoretical knowledge, the study group that used videos presented superior performance in the evaluation ( $p<$ 0.041). Conclusion: The two pedagogical tools both enhanced the students' knowledge, where the group that used videos showed deeper theoretical knowledge of the procedure, but procedure execution was identical between groups. 


\section{INTRODUÇÃO}

Com o advento da tecnologia, as instituições de ensino são desafiadas a criar novas metodologias pedagógicas para seus alunos e professores, propondo, além de aulas teóricas, aulas práticas, materiais impressos, recursos da internet e uso de vídeos educacionais para melhorar a satisfação, motivação e retenção de conhecimento do aluno, além de otimizar o tempo de estudo ${ }^{1,2,3}$.

Novos recursos contribuem para a formação educacional, pois propiciam melhor aprendizado das situações criadas e dos ambientes desenvolvidos, além de melhorar a capacidade de interação dos alunos. Diante de recursos como animação, cores e sons, é importante o conhecimento amplo sobre a teoria da carga cognitiva referente à maneira como os seres humanos captam, codificam, estocam e recuperam as informações ${ }^{4,5}$.

Ha algumas décadas, já se cogitava usar materiais audiovisuais no contexto educativo, pois se acreditava que filmes e vídeos, dotados de uma linguagem universal e multissensorial, forneciam uma comunicação superior à das palavras ${ }^{6}$.

A introdução de novas tecnologias e métodos de ensino associados a vídeos tem contribuído para aprimorar e permitir este aprendizado de maneira mais precoce, garantindo a segurança dos pacientes. Uma destas novas metodologias é a Simulação Médica, que, além de permitir a exposição repetida segura, expõe estudantes a inúmeras situações da prática profissional e contribui para aperfeiçoar habilidades não técnicas, tais como comunicação, trabalho em equipe, colaboração, envolvimento e tomada de decisão ${ }^{7,8,9}$.

Alguns estudos de aplicações multimídias observaram que a utilização inadequada da percepção, visão e audição pode desorientar e até desestimular o aluno, principalmente quando o material emprega vários recursos simultâneos, como sons, imagens e narração ${ }^{10}$.

O presente estudo objetivou identificar a melhor metodologia para retenção do conhecimento teórico e prático dos estudantes de Medicina no treinamento de entubação orotraqueal, comparando o checklist com o vídeo educacional, além de somar outra proposta pedagógica para os professores e auxiliar no estudo individualizado dos alunos.

\section{METODOLOGIA}

\section{Produção}

Os vídeos foram produzidos no laboratório de Simulação em Saúde da própria universidade, utilizando equipamentos médicos reais e/ou simulados, manequins que mimetizam funções vitais humanas e os próprios autores, que representaram pacientes para a execução de algumas técnicas semiológicas.
Na filmagem, foi empregada uma filmadora digital com tripé, e a edição foi feita em um computador pessoal. Tanto a filmagem quanto a edição dos vídeos foram executadas pelos alunos autores do projeto e colaboradores.

Os temas foram escolhidos pela pertinência da importância para alunos que estão em fase de aprendizado de procedimentos médicos. Os vídeos escolhidos para gravação e edição estão listados no Quadro 1.

QuAdro 1

Temas selecionados para a confecção dos vídeos

\begin{aligned} & \hline 1 Controle de sinais vitais \\ & \hline 2 Aferição da pressão arterial não invasiva \\ & \hline 3 Sonda nasogástrica \\ & \hline 4 Cateterismo vesical \\ & \hline 5 Aspiração orotraqueal \\ & \hline 6 Punção venosa periférica \\ & \hline 7 Entubação orotraqueal \\ & \hline 8 Entubação com máscara laríngea \\ & \hline 9 Oxigenoterapia não invasiva \\ & \hline 10 Parto normal \\ & 11 RCP-DEA-adulto \\ &\end{aligned}

\section{Amostra}

O estudo foi realizado no Campus Centro da Universidade Anhembi Morumbi, situada à Rua Dr. Almeida Lima, 1134, em São Paulo. A coleta de dados foi realizada pelos pesquisadores envolvidos neste projeto. Foram estudados alunos do quarto semestre do curso de Medicina dessa universidade.

\section{Procedimento}

Foram selecionados aleatoriamente 30 alunos do curso de Medicina matriculados no quarto semestre. A avaliação ocorreu no laboratório de Simulação em Saúde e abordou o tema Entubação Orotraqueal.

Os alunos foram submetidos a uma avaliação teórica e prática sobre o assunto para mensurar o conhecimento após um ano da ministração da disciplina conforme a grade curricular (avaliação inicial). Imediatamente após as provas, os participantes foram randomizados em dois grupos, A e B. O grupo A recebeu o vídeo referente ao assunto, e o grupo $\mathrm{B}$ teve acesso somente ao checklist (descrição detalhada de todas as etapas do procedimento) para o estudo.

Após terem cumprido uma carga de estudo de duas horas obrigatórias durante uma semana, todos foram avaliados novamente com a mesma prova teórica e prática após sete dias. 
Os resultados das avaliações foram analisados com relação ao aprendizado.

\section{ANÁLISE ESTATÍSTICA}

A análise dos resultados obtidos foi realizada em primeiro momento intragrupos, para verificar se os alunos adquiriram conhecimento com as estratégias de ensino adotadas, e, em segundo momento, entre os grupos, com o objetivo de comparar os métodos de ensino e sua eficácia.

Inicialmente, analisou-se a nota obtida nas provas iniciais de cada grupo (A e B) através do teste $t$ para amostras não pareadas, com o objetivo de verificar a homogeneidade da amostra. Em seguida, procedeu-se à comparação entre as notas iniciais e finais da avaliação teórica e o número de acertos da prova prática de cada grupo através do teste $t$ para amostras pareadas.

Para verificar se houve diferença na variação de resultados entre os grupos (comparação de média entre grupos), utilizou-se o teste $\mathrm{t}$ para amostras não pareadas.

Os resultados foram considerados significativos quando atingiram nível de significância de $\mathrm{p}<0,05$.

\section{RESULTADOS}

Foram selecionados aleatoriamente 30 alunos do curso de Medicina matriculados no quarto semestre, divididos em grupos A e B. A seguir, descrevem-se separadamente os resultados obtidos nas avaliações teóricas e práticas de cada grupo.

\section{Avaliação teórica}

Para verificar a homogeneidade de conhecimentos teóricos prévios, foram comparadas as avaliações teóricas realizadas no dia da randomização (Tabela 1). Observou- se que não houve diferença nos conhecimentos iniciais entre os grupos ( $\mathrm{p}=$ 0,189).

\begin{tabular}{ccccc} 
Homogeneidade da amostra & \\
Grupo & $\begin{array}{c}\text { Média } \\
\text { inicial }\end{array}$ & $\begin{array}{c}\text { Desvio } \\
\text { padrão }\end{array}$ & $\begin{array}{c}\text { Intervalo de } \\
\text { confiança (95\%) }\end{array}$ & p \\
\hline A & 6,46 & 0,56 & $-0,51-0,11$ & 0,189 \\
B & 6,67 & & & \\
\hline
\end{tabular}

Após uma semana de estudo com uma carga de duas horas, cada grupo foi submetido a uma avaliação teórica final. Os resultados indicam que houve ganho de conhecimento em ambos os grupos, mas aquele que estudou por vídeos obteve melhor nota na avaliação final (Tabela 2).

\begin{tabular}{cccccc}
\multicolumn{7}{c}{ TABELA 2} \\
Comparação da variação das notas para cada grupo
\end{tabular}

Ao se comparar a evolução das notas entre os grupos, observou-se que os alunos que estudaram por meio de vídeos obtiveram melhor desempenho na avaliação teórica $(\mathrm{p}<$ 0,041), o que demonstra maior efetividade do uso de imagens para o ganho de conhecimento quando comparado ao material impresso para leitura (Tabela 3).

\begin{tabular}{|c|c|ccc|}
\hline \multicolumn{5}{c|}{ TABELA 3} \\
Comparação da variação das notas entre os grupos A e B \\
\hline Grupo & $\begin{array}{c}\text { Média } \\
\text { final }\end{array}$ & $\begin{array}{c}\text { Desvio } \\
\text { padrão }\end{array}$ & $\begin{array}{c}\text { Intervalo de } \\
\text { confiança (95\%) }\end{array}$ & p \\
\hline A & 8,93 & & & \\
B & 8,40 & 0,91 & $0,03-1,04$ & 0,041 \\
\hline
\end{tabular}

Sendo assim, observou-se neste ensaio que houve ganho de conhecimento para todos os alunos, porém aqueles randomizados para estudo por meio de material audiovisual apresentaram melhor desempenho na avaliação teórica (Figura 1).

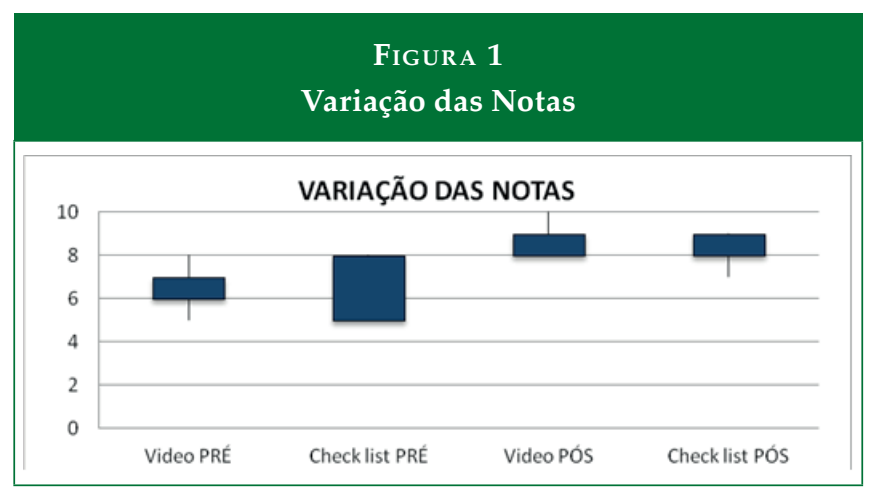

\section{Avaliação prática}

Ao se analisarem separadamente as respostas das provas práticas de cada grupo, verificou-se que o conhecimento inicial foi semelhante entre os grupos $(p=0,73)$, garantindo a homogeneidade da amostra. Após treinamento, nota-se que o grupo A (vídeo) apresentou pior resultado em cinco questões: conexão do ambu na fonte de oxigênio, ventilação adequada após entubação, familiaridade no manejo do laringoscópio, 
insuflação do cuff e entubação em local inadequado, porém todos encontraram o erro imediatamente e corrigiram a posição da cânula.

Por outro lado, o grupo B apresentou pior desempenho em sete itens avaliados: lavagem das mãos antes do procedimento, colocação de fio-guia no tubo em posição adequada, abertura de vias aéreas no momento da ventilação, inserção do tubo traqueal na posição correta, retirada do fio-guia, apoio da cânula até sua fixação e verificação de ventilação eficaz após a entubação. Apesar das diferenças de desempenho entre os grupos, o resultado não apresentou significância $(p=1)$, demonstrando que o conhecimento prático foi idêntico ou até mesmo que houve interferência de fatores emocionais, como ansiedade, pela realização de avaliação prática.

Ao se comparar o conhecimento adquirido pelos grupos, nota-se que ambos melhoraram seus conhecimentos (grupo A: $\mathrm{p}<0,012$ e grupo B: $\mathrm{p}<0,08)$. Assim, observa-se que todos melhoraram seu desempenho prático, mas, apesar do treinamento e material oferecido, ainda permanecem falhas no aprendizado, embora o grupo de treinamento por meio de material audiovisual tenha apresentado melhor resultado e preparo na execução do procedimento.

\section{DISCUSSÃO}

Um estudo realizado em universidade acadêmica nos diferentes setores de terapia intensiva (Pneumologia, Anestesiologia e Pronto-Socorro) avaliou o conhecimento prévio dos profissionais por meio de questionário, com itens relacionados ao grau de conhecimento da técnica de entubação orotraqueal, e verificou que existem divergências entre a prática médica e a literatura, as quais podem ocasionar iatrogenias. O estudo verificou desde manobras iniciais, como pré-oxigenação, utilização de coxim, manobra de sellick e uso de drogas específicas na indução do paciente, até o tempo de experiência do profissional médico. Concluiu-se que o conhecimento em entubação orotraqueal é insatisfatório. Por isso, é importante reforçar o estudo sobre o tema na formação médica e criar uma nova pedagogia de ensino para fortalecer o conhecimento e evitar possíveis complicações no procedimento, como entubação esofágica ou seletiva, barotrauma, trauma de vias aéreas superiores e dos dentes, e arritmias cardíacas, entre outros ${ }^{11}$.

Pode-se observar que houve maior ganho de conhecimento na prova teórica e menor quantidade de erros na avaliação prática no grupo que utilizou o vídeo educacional. Em estudo feito na Universidade de Auckland, Nova Zelândia, foi apresentado a um grupo de médicos em treinamento um vídeo sobre cateterismo vesical em homens e mulheres. O grupo de 11 participantes que teve acesso ao vídeo foi comparado a ou- tro grupo de 10 participantes que não teve acesso ao vídeo. Ambos os grupos receberam treinamento com simulação da sondagem vesical em manequins.

Todos os participantes praticaram a sondagem, sendo avaliados pelo mesmo corpo docente. Após a avaliação, os participantes completaram novamente o questionário sobre os níveis de confiança e habilidade. Nos três meses subsequentes, apenas um dos grupos teve acesso ao vídeo, e o outro grupo não recebeu nenhuma intervenção educacional. No final de três meses, os participantes repetiram a avaliação prática no laboratório e o questionário. Observou-se que o grupo que teve acesso aos vídeos obteve maiores índices de competência e autoconfiança, especialmente para a sondagem vesical feminina ${ }^{10}$.

Vídeos médicos já vêm sendo empregados em treinamentos, e alguns autores acreditam que a associação dos vídeos no momento do treinamento pode ser ainda mais produtiva, melhorando o desenvolvimento psicomotor e a retenção, no mesmo modelo do Watch then Practice, amplamente utilizado nos treinamentos de ressuscitação cardiopulmonar da American Heart Association, uma das pioneiras no uso deste tipo de técnica ${ }^{6,10,12}$.

Além dos vídeos, outra ferramenta para auxiliar o estudo individualizado é o checklist, um guia que descreve determinado procedimento passo a passo, ajudando no estudo individualizado. Atualmente é aplicado em diversas instituições para orientar o aluno, como utilizado nesta pesquisa, e comparado com o vídeo, que apresenta legendas e imagens, auxilia a aprimorar o manejo adequado do material, melhora a habilidade e corrige erros, o que não ocorre no checklist porque este contém somente palavras.

Hoje podemos observar em alguns trabalhos que os alunos estão sendo os protagonistas e roteiristas da educação médica, ou seja, elaboram e aperfeiçoam matérias didáticas para somar valores e propõem novas abordagens de ensino junto com os professores ${ }^{1,9,13}$

\section{CONCLUSÃO}

Nas duas ferramentas pedagógicas (vídeo educacional e checklist), houve ganho de conhecimento por parte do aluno, principalmente no conhecimento teórico daqueles que estudaram por vídeos. Alguns erros ainda foram identificados, e, apesar do treinamento e material didático oferecido, ainda permanecem falhas no aprendizado.

\section{REFERÊNCIAS}

1. Vasconcelos DFP, Vasconcelos ACCG. Desenvolvimento de um Ambiente Virtual de Ensino em Histologia para Es- 
tudantes da Saúde. Revista Brasileira de Educação Médica. 2013;37(1):132-137.

2. Lampert JB. Currículo de Graduação e o Contexto da Formação do Médico. Rev Bras Educ Méd. 2000; 24(3):7-19.

3. Braccialli LAD, Oliveira MAC. Desafios na Formação Médica: a Contribuição da Avaliação. Revista Brasileira de Educação Médica. 2012; 36(2):280-268.

4. Santos LMA, Tarouco LMR. A importância do Estudo da Teoria da Carga Cognitiva em uma Educação Tecnológica. Novas Tecnologias CINTED-UFRGS na Educação 2007;5(1).

5. Nunes M, Giraffa L. A educação na ecologia digital. Porto Alegre: PUCRS; 2003.

6. Worth S. The Uses of Film in Education and Communication. In: David RO, ed. Symbols: The forms of the expression, comunication, and educations, Part I, Seventy-third yearbook of the National Society for the Study of Education. Chicago: University of Chicago Press; 1979.

7. Grant DJ, Marriage SC. Training using medical simulation. Arch. Dis. Child. 2011.

8. Ziv A, Wolpe PR, Small SD, Glick S. Simulation-based medical education: an ethical imperative. Simul Healthc. 2006;1(4):252-6.

9. Arruda FT, Danek A, Abrão KC, Quilici AP. Elaboração de vídeos médicos educacionais para treinamento de habilidades de estudantes do curso de medicina. Rev. bras. educ. med. 2012;36(3).

10. Mayer R. Multimedia Learning. Cambridge: Cambridge University Press.2001.

11. Yamanaka CS, Góis AFT, Vieira PCB, Alves JCD, Oliveira LM, Blanes L, Lourenço EPL, Assunção M, Machado FR. Intubação orotraqueal: avaliação do conhecimento médico e das práticas clínicas adotadas em unidades de terapia intensiva. Rev Bras Ter Intensiva. 2010; 22(2):103-111.

12. American Heart Association Guidelines 2005 for Cardiopulmonary Ressuscitation and Emergency Cardiovascular Care. Circulation. 2005; 112: 4-19.

13. Junior AAP, Rezende LAC, Bastos WG. Recepção Audiovisual na Educação Médica: Leituras de um Vídeo Educativo de Psicologia Médica por Estudantes de Medicina. Revista Brasileira de Educação Médica. 2012; 36(4):516-523.

\section{CONTRIBUIÇÃO DOS AUTORES}

Allan Danek e Felipe Teles de Arruda realizaram as gravações dos vídeos, a supervisão do estudo individualizado e aplicaram as provas prática e teórica. Ana Paula Quilici orientou o trabalho e auxiliou na correção do projeto.

\section{COLABORADORES}

Profa. Elaine Peixoto, Prof. Dr. Robert Fabian Crespo Rosas, Dra. Gabriela Von Wincler e Profa. Raquel Ferraz auxiliaram na estrutura e apoio logístico do trabalho.

\section{CONFLITO DE INTERESSES}

Declarou não haver.

\section{ENDEREÇO PARA CORRESPONDÊNCIA}

Allan Danek

Rua Almirante Noronha 956 - apto 94

Jd. São Paulo - São Paulo

CEP 02043-061 - SP

E-mail:danekkk@hotmail.com 